\title{
Climate Change Impact on Quiver Trees in Arid Namibia and South Africa
}

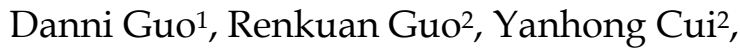 \\ Guy F. Midgley ${ }^{1}$, Res Altwegg ${ }^{1}$ and Christien Thiart ${ }^{2}$ \\ ${ }^{1}$ Climate Change and Bioadapation Division \\ South African National Biodiversity Institute, Cape Town \\ ${ }^{2}$ Department of Statistical Sciences, University of Cape Town, Cape Town \\ South Africa
}

\section{Introduction}

The climate fluctuates and changes naturally, and adding the common problems of land transformation and deforestation, its impact can be very harsh on the natural environment, and cause a decline in the biodiversity of plants and animals. Aloe dichotoma, common name Quiver tree, is an important part of the arid regions, such as Namaqualand and Bushmanland in South Africa, and in arid parts of southern Namibia. This succulent tree species occurs in rocky areas, and it can grow quite rapidly under the right conditions. Succulents are able to survive long periods of drought conditions, due to the fact succulent plants has special water-storing tissue which makes part of the plant fleshy, and the Quiver tree has succulent leaf and stem (Van Wyk and Smith, 1996).

The Quiver tree has a 200 year life span, and can grow up to 9 meters tall, and it occurs in summer and winter rainfall regions, and can live under a variety of climatic conditions (Fig. 1). The Quiver tree is important to the ecosystem due to the fact that it is as a source of moisture for a wide variety of mammals, birds, and insects. Foden's detailed study of the demographic data of the Quiver trees show that negligible recruitment has occurred in certain populations for 50 years, and the effects of non-climatic variables, such as herbivory, competition, seed availability, fungal pathogens, plant collection... are very small (Foden, 2002).

Today, the Quiver trees are threatened by agricultural expansion, overgrazing, and mining, as well as droughts and other climate changes (Foden, 2002). Climate changes is one of the major factors affecting the existence of Quiver trees, while the Quiver tree will unlikely to be affected by small climatic fluctuations, but will be affected larger or long term climatic changes. The Quiver tree can potentially provide a good indication of long term climate changes in the arid regions (Foden, 2002).

Previous onsite observations show that Quiver trees are very sensitive to temperature changes, and does not do well under extreme hot and dry conditions. Observations has also shown that the Quiver trees might be responding to higher temperatures by shifting its distribution range towards higher and higher altitudes, showing a preference for slightly cooler regions (Midgley et al., 2009). 


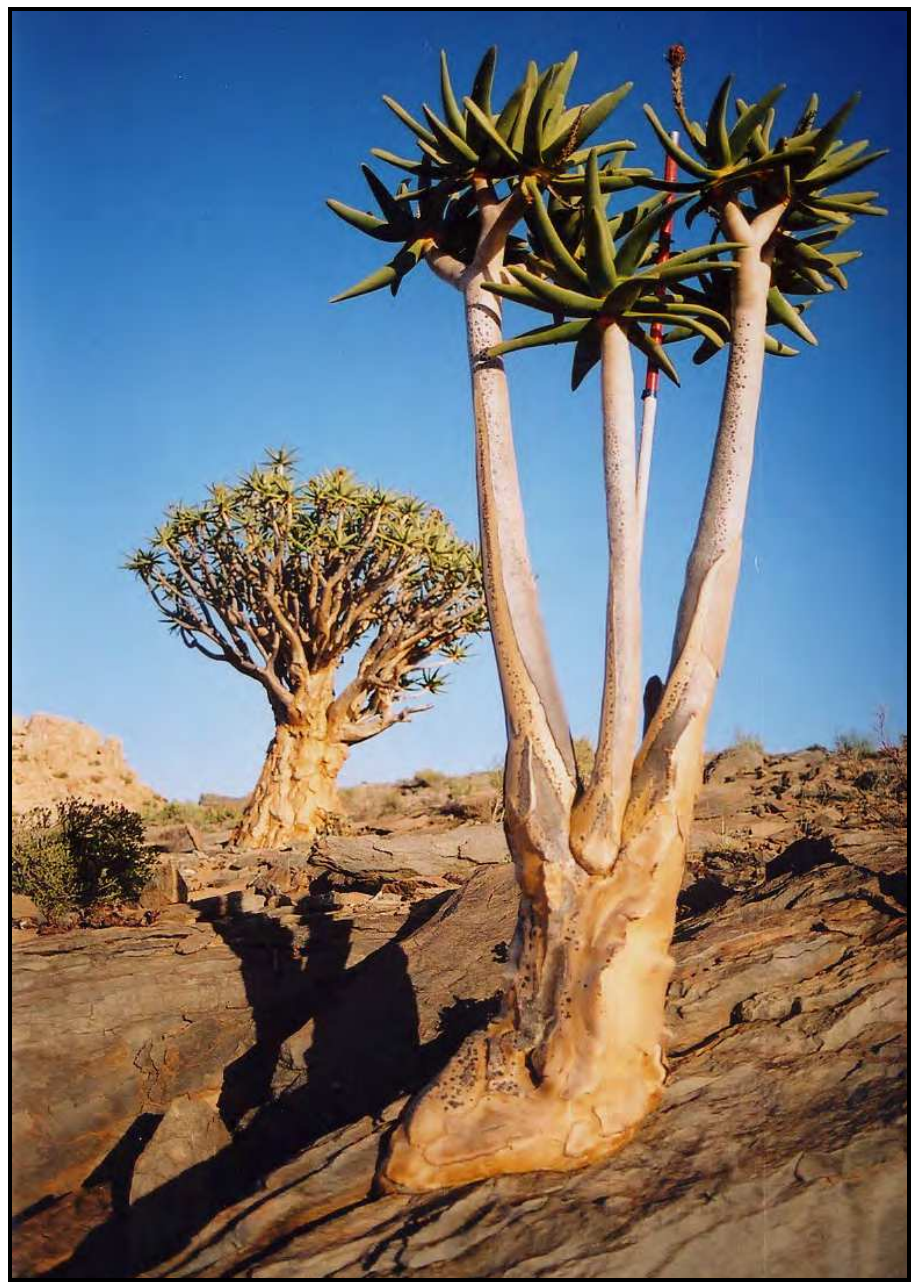

Fig. 1. Quiver trees in Kaukusib Koppie, Namibia, 2008

To have a complete picture of the Quiver tree's response to recent climatic changes, samples of Quiver trees were collected from their distributional range in arid Namibia and South Africa, and this is a continuing project funded by the South African National Biodiversity Institute. However, data uncertainty accompanied the sample collections as well.

In 2001 and 2002, Wendy Foden and various assistants collected Quiver trees sample data across the whole range, sampling 53 sites in detail. In 2008 Graeme Ellis re-sampled a subset of the population, sampling 41 sites. In 2009, Res Altwegg and Cory Merow did a thorough analysis and comparison of between the 2 sets of data, for 35 sites, excluding certain sites due to lack of data for comparisons.

In this chapter, we are going one step further, that is, to try to complete all the missing data values, which will allow us to do a more complete comparison. The incomplete data issue is complicated on many fronts: 
1. While the 2001-2002 data are the most complete, however, there are several sites with missing variables from the 53 sample sites.

2. The 2008 data is a subset of the original 2001-2002 data, covering 41 sites, and the 2008 data variables are not the same as the 2001-2002 data variables.

3. The 2009 analysed data comparison between 2001-2002 and 2008 data is for 35 sites, with 18 sites uncalculated due to missing data.

4. With all 3 datasets, missing data is a major part of the problem, which renders the 3 datasets incomplete and only partially comparable.

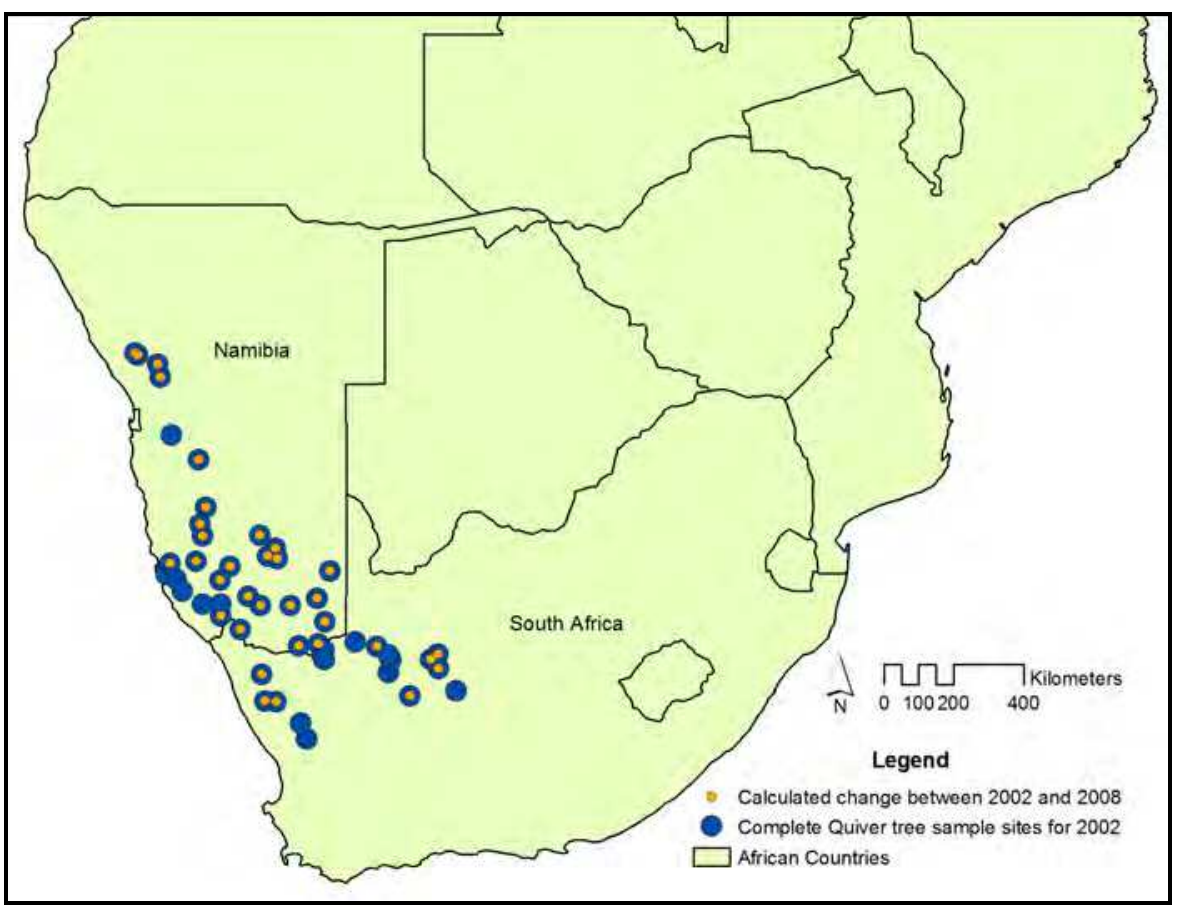

Fig. 2. Available Quiver tree sample site data in Arid Namibia and South Africa

Of course, another sample collection study would be ideal, but to due lack of funding and trained professionals, this is not possible at this stage. In any case, a full usage should be made of the 2002 and 2008 data. In Fig. 2, the Quiver trees sample sites are shown, the Quiver tree's distribution extending over the arid regions in Namibia and South Africa. As one can see from the figure, the 2008 study is a subset of the original 2002 samples.

\section{Impreciseness in quiver tree's sample data}

To investigate the climate change impacts on the Quiver's spatial distribution from 2002 to 2008, we must have the Quiver's population evolution information. We as biodiversity scientists need to reveal the climate change impacts on plant species based on the data collected. Only solid data-oriented analysis can provide near-true information to the public and governmental decision-making body. In this case, the Quiver tree's dataset is not 
complete enough to perform a solid data-oriented analysis because of the impreciseness features of the dataset.

Impreciseness is a fundamental and intrinsic feature in a solid data oriented spatial modelling exercises due to the observational data shortage and incompleteness. Facing the impreciseness reality the spatial statisticians often rely on expert's knowledge to compensate the inadequacy and in accuracy in collected observational data. Nevertheless, such a dependence on experts' knowledge engineering will still open a backdoor to pseudoscientific believe or claim.

Impreciseness is referred to a term with an intrinsic property governed by an uncertain measure or an uncertainty distribution for each of the actual or hypothetical members of an uncertainty population (i.e., collection of expert's knowledge). An uncertainty process is a repeating process whose outcomes follow no describable deterministic pattern, but follow an uncertainty distribution, such that the uncertain measure of the occurrence of each outcome can be only approximated or calculated.

Definition 2.1: Impreciseness is an intrinsic property of a variable or an expert's knowledge being specified by an uncertainty measure.

In geo-statistics analyst communities it is seldom to mention uncertainty measure. It is true, spatial researchers are used to the term "uncertainty", which represents randomness in their eyes. Actually, this perception is wrong. Researchers have long realized the there are many forms of uncertainty, randomness is merely a member of uncertainty family. How can we differentiate different forms of uncertainty? The answer is fairly straght forward: the appropriate measure specifies a particular form of uncertainty. For example, probability measure specifies random uncertainty.

The uncertainty modelling without a measure specification will not have an rigorous mathematical foundations and consequently the modelling exercise is baseless and blindness. In other words, measure specification is the prerequisite to spatial data collection and analysis. For example, without Kolmogrov's (1950) three axioms of probability measure, randomness is not defined and thus statistical data analysis and inference has no foundation at all.

Notice that imprecise probability theory is a potential remedy to address the observational data inaccuracy and inadequacy because most the geo-statistics analysts are familiar with probability theory. However the imprecise probability based spatial modelling might be not feasible. Just as Utikin and Gurov (2000) has commented, "the probabilistic uncertainty model makes sense if the following three premises are satisfied: (i) an event is defined precisely; (ii) a large amount of statistical samples is available; (iii) probabilistic repetitiveness is embedded in the collected samples. This implies that the probabilistic assumption may be unreasonable in a wide scope of cases."

It is therefore inevitably to seek another form of uncertainty theory to meet the impreciseness challenges. In the theoretical basket, interval uncertainty theory (Moore, 1966), fuzzy theory (Zadeh, 1965, 1978), grey theory (Deng, 1984), rough set theory (1982), upper and lower provisions or expectations (Walley, 1991), or Liu's uncertainty theory $(2007,2010)$ may be chosen. Nevertheless, Liu's $(2007,2010)$ uncertainty theory is the only one built on an axiomatic uncertain measure foundation and fully justified with mathematical rigor. Therefore it is logical to engage Liu's $(2007,2010)$ uncertainty theory for guiding us to understand the intrinsic character of imprecise uncertainty and facilitate an accurate mathematical definition of impreciseness in order to establish the foundations for uncertainty spatial modelling under imprecise uncertainty environments. 


\section{Uncertain measure foundation}

The Uncertainty Theory was founded by Liu in 2007 and refined in 2010 (Liu, 2007, 2010). Nowadays uncertainty theory has become a branch of mathematics.

A key concept in uncertainty theory is the uncertain measure: Let $\Xi$ be a nonempty set (space), and $\mathfrak{A}(\Xi)$ the $\sigma$-algebra on $\Xi$. Each element, let us say, $A \subset \Xi, A \in \mathfrak{A}(\Xi)$ is called an uncertain event. A number denoted as $\lambda\{A\}, 0 \leq \lambda\{A\} \leq 1$, is assigned to event $A \in \mathfrak{A}(\Xi)$, which indicates the uncertain measuring grade with which event $A \in \mathfrak{A}(\Xi)$ occurs. The normal set function $\lambda\{A\}$ satisfies following axioms given by Liu (2011):

Axiom 1: (Normality) $\lambda\{\Xi\}=1$.

Axiom 2: (Self-Duality) $\lambda\{\cdot\}$ is self-dual, i.e., for any $A \in \mathfrak{A}(\Xi), \lambda\{A\}+\lambda\left\{A^{c}\right\}=1$.

Axiom 3: ( $\sigma$-Subadditivity) $\lambda\left\{\bigcup_{i=1}^{\infty} A_{i}\right\} \leq \sum_{i=1}^{\infty} \lambda\left\{A_{i}\right\}$ for any countable event sequence $\left\{A_{i}\right\}$.

Definition 3.1: (Liu, 2007, 2010) A set function $\lambda: \mathfrak{A}(\Xi) \rightarrow[0,1]$ satisfies Axioms 1-4 is called an uncertain measure. The triple $(\Xi, \mathfrak{A}(\Xi), \lambda)$ is called an uncertainty space.

Definition 3.2: (Liu, 2007, 2010) An uncertain variable is a measurable function $\xi$ from an uncertainty space $(\Xi, \mathfrak{A}(\Xi), \lambda)$ to the set of real numbers.

Definition 3.3: (Liu, 2010) Let $\xi$ be a uncertainty quantity of impreciseness on an uncertainty measure space $(\Xi, \mathfrak{A}(\Xi), \lambda)$. The uncertainty distribution of $\xi$ is

$$
\Psi_{\xi}(x)=\lambda\{\tau \in \Xi \mid \xi(\tau) \leq x\}
$$

Theorem 3.4: (Liu, 2007, 2010) Let $\Psi_{\xi_{1}}, \Psi_{\xi_{2}}, \cdots, \Psi_{\xi_{n}}$ be uncertainty distributions for the uncertainty variables $\xi_{1}, \xi_{2}, \cdots, \xi_{n}$ on $(\Xi, \mathfrak{A}(\Xi), \lambda)$ respectively. Let $\Psi_{\left(\xi_{1}, \xi_{2}, \cdots, \xi_{n}\right)}$ be the joint distribution of uncertainty vector $\left(\xi_{1}, \xi_{2}, \cdots, \xi_{n}\right)$. If $\xi_{1}, \xi_{2}, \cdots, \xi_{n}$ are independent, then

$$
\Psi_{\left(\xi_{1}, \xi_{2}, \cdots, \xi_{n}\right)}\left(x_{1}, x_{2}, \cdots, x_{n}\right)=\min _{1 \leq i \leq n} \Psi_{\xi_{i}}\left(x_{i}\right)
$$

for any real numbers $x_{1}, x_{2}, \cdots, x_{n} \in \mathbb{R}$.

Definition 3.5: (Liu, 2007, 2010) Let $\xi$ be a uncertainty variable on an uncertainty measure space $(\Xi, \mathfrak{A}(\Xi), \lambda)$. The expectation $\xi$ is defined by

$$
\mathrm{E}[\xi]=\int_{0}^{+\infty} \lambda\{\xi \geq r\} d r-\int_{-\infty}^{0} \lambda\{\xi \leq r\} d r
$$

provided that one of the two integrals exists at least.

Definition 3.6: (Liu, 2007, 2010) Let $\xi$ be a uncertainty variable on an uncertainty measure space $(\Xi, \mathfrak{A}(\Xi), \lambda)$.with uncertainty distribution function and $\Psi_{\xi}$ a finite expectation $\mu$. Then the variance of $\xi$, denoted by $V[\xi]$, is defined by

$$
V[\xi]=2 \int_{0}^{+\infty}(r-\mu)\left(1-\Psi_{\xi}(r)+\Psi_{\xi}(2 \mu-r)\right) d r .
$$

Liu's uncertainty theory (Liu, 2007, 2010) does not define multivariate uncertainty distribution, (Guo, 2010; Guo et al., 2007), except for the independent uncertainty variables. 
Hence it is necessary to facilitate a framework for showing the preparation to define the uncertain covariance.

Let $\Psi_{\xi_{1}}, \Psi_{\xi_{2}}, \cdots, \Psi_{\xi_{n}}$ be uncertainty distributions for the uncertainty variables $\xi_{1}, \xi_{2}, \cdots, \xi_{n}$ on $(\Xi, \mathfrak{A}(\Xi), \lambda)$ respectively. Let $\Psi_{\left(\xi_{1}, \xi_{2}, \cdots, \xi_{n}\right)}$ be the joint distribution of uncertainty vector $\left(\xi_{1}, \xi_{2}, \cdots, \xi_{n}\right)$. Assuming that $\xi_{i}$ and $\xi_{j}$ two arbitrary pair of uncertainty variables within the uncertainty vector $\left(\xi_{1}, \xi_{2}, \cdots, \xi_{n}\right)$ which have finite expectations $\mu_{1}$ and $\mu_{2}$ respectively. Denote $\Psi_{\left(\xi_{i}, \xi_{j}\right)}$ bivariate uncertainty distribution function.

Definition 3.7: Let $\eta_{i j}=\left(\xi_{i}-\mu_{i}\right)\left(\xi_{j}-\mu_{j}\right)$ be product of centered uncertainty variables $\xi_{i}$ and $\xi_{j}$. The uncertainty distribution of $\eta_{i j}$ is defined by

$$
\Psi_{\eta_{i j}}(y)=\sup _{z_{1} z_{2}=y} \Psi_{\left(\xi_{i}, \xi_{j}\right)}\left(z_{i}, z_{j}\right), \forall\left(z_{i}, z_{j}\right) \in \mathbb{R}^{2}
$$

Definition 3.8: Let $\eta_{i j}=\left(\xi_{i}-\mu_{i}\right)\left(\xi_{j}-\mu_{j}\right)$ be product of centered uncertainty variables $\xi_{i}$ and $\xi_{j}$. The expectation of centered product $\eta_{i j}$ is called the covariance between uncertainty variables $\xi_{i}$ and $\xi_{j}$, that is by

$$
\gamma_{i j} \triangleq \mathrm{E}\left[\eta_{i j}\right]=\int_{0}^{+\infty}\left(1-\Psi_{\eta_{i j}}(r)\right) d r-\int_{-\infty}^{0} \Psi_{\eta_{i j}}(r) d r
$$

Theorem 3.9: Let $\xi_{1}, \xi_{2}, \cdots, \xi_{n}$ be independent uncertainty variables on $(\Xi, \mathfrak{A}, \boldsymbol{\lambda})$. Then

$$
\Psi_{\eta_{i j}}(y)=\sup _{z_{1} z_{2}=y}\left(\Psi_{\xi_{1}}\left(z_{1}\right) \wedge \Psi_{\xi_{2}}\left(z_{2}\right)\right), \forall\left(z_{i}, z_{j}\right) \in \mathbb{R}^{2}
$$

Remark 3.10: Different from independent random variables $X_{1}$ and $X_{2}$, whose $\mathrm{E}\left[\left(X_{1}-\mathrm{E}\left[X_{1}\right]\right)\left(X_{2}-\mathrm{E}\left[X_{2}\right]\right)\right]=0$. In uncertainty theory, independent $\xi_{1}, \xi_{2}, \cdots, \xi_{n}$ do not imply $\eta_{i j}=0$.

Remark 3.11: The formation of uncertain variance-covariance does touch the detailed functional form of multivariate uncertainty joint distribution. For practical applications, the form of multivariate uncertainty joint distribution is not necessarily available, but the paired uncertainty bivariate distribution must be given.

Similar to the concept of stochastic process in probability theory, an uncertain process $\left\{\xi_{t}, t \geq 0\right\}$ is a family of uncertainty variables indexed by $t$ and taking values in the state space $\mathbb{S} \subseteq \mathbb{R}$.

Definition 3.12: (Liu, 2007, 2010) Let $\left\{C_{t}, t \geq 0\right\}$ be an uncertain process.

(1) $C_{0}=0$ and all the trajectories of realizations are Lipschitz-continuous;

(2) $\left\{C_{t}, t \geq 0\right\}$ has stationary and independent increments;

(3) every increment $C_{t+s}-C_{s}$ is a normal uncertainty variable with expected value 0 and variance $t^{2}$, i.e., the uncertainty distribution of $C_{t+s}-C_{s}$ is

$$
\Psi_{C_{t+s}-C_{s}}(z)=\left(1+\exp \left(-\frac{\pi z}{\sqrt{3} t}\right)\right)^{-1}
$$

then $\left\{C_{t}, t \geq 0\right\}$ is called an uncertain canonical process. 
Theorem 3.13: Assuming that $\left\{C_{t}, t \geq 0\right\}$ is an uncertain canonical process. Therefore, in the autocovariance and autocorrelation of uncertain canonical process $\left\{C_{t}, t \geq 0\right\}$ are

$$
\sigma_{s, t}=s^{2}+\mathrm{E}\left[C_{s}\left(C_{t}-C_{s}\right)\right]=s^{2}+\delta_{s, t-s}
$$

and

$$
\rho_{s, t}=\frac{s}{t}+\frac{\delta_{s, t-s}}{s t},(s<t)
$$

where

$$
\begin{aligned}
& \delta_{s, t-s}=E\left[C_{s}\left(C_{t}-C_{s}\right)\right] \\
& =\int_{0}^{\infty}\left(1-\sup _{x y=r}\left(\Psi_{C_{s}}(x) \wedge \Psi_{C_{t-s}}(y)\right)\right) d r \\
& -\int_{-\infty}^{0} \sup _{x y=r}\left(\Psi_{C_{s}}(x) \wedge \Psi_{C_{t-s}}(y)\right) d r
\end{aligned}
$$

\section{Habitat measure}

Note that the Quiver tree's 2002 data subset contains percentage of dead trees, percentage of juvenile trees, the total density of trees, and the average age of dead trees. Also, Quiver tree's 2008 data subset contains population growth rate, survival reproductives, survival non-reproductives, and proportion juvenile. Considering the aim of this research: climate change impacts on the Quiver tree's population, we select total density of tree (in 2002 data subset) and population growth rate (in 2008 data subset) as the our analysis data. We will build our model based on the total tree population size

$$
V_{i}^{(k)}=A^{(k)} \kappa_{i}^{(k)}
$$

where the $V_{i}^{(k)}$ is the total population size at year $i$ over area size $A^{(k)}$, and total density $\kappa_{i}^{(k)}$.

Notice that among 53 sites, 43 sites have total density observational values. Ten sites have missing values. That is, without total density observation $\{10,12,14,15,17,19,33,36,37,128\}$ site number set.

For inexperienced go-statistics analysts, kriging method might be a choice for filling the ten missing vaules. Nevertheless, after examining the 2002 total density of tree's 43 observations, we found that geometric distance does not play a role in dertermining the value of the total density of trees. we can find out that the total density observations are not similar even for the very nearby sites geometrically within the same ecological sub-region. For example, Site, see Table 1.

From Table 1, it is obvious that for the Quiver tree's growth status, $\left\{Z(s), s \in \mathbb{R}^{2}\right\}$, the spatial location $s \in \mathbb{R}^{2}$ only partially links to the tree habitation. While the geometric distance between two locations $s$ and $s+h$ is no longer fully reflecting the habitat closeness. The Quiver tree's growth status observations reveal a fact that the total density status is extremely sensitive to the ecological conditions of individual site area (or the area habitat). 
The graphical location and the isotropic distance will not offer much information, rather the Quiver tree's growth observation $Z(s)$ gives the best indication of the tree area habitat.

\begin{tabular}{|c|c|c|c|c|c|c|c|}
\hline \multirow{2}{*}{\multicolumn{2}{|c|}{$\begin{array}{l}\text { Group/ } \\
\text { site no. }\end{array}$}} & \multicolumn{2}{|c|}{ Coordinates } & \multirow{3}{*}{\begin{tabular}{|l|}
$\begin{array}{l}\text { Total } \\
\text { density of } \\
\text { trees }\end{array}$ \\
36.41 \\
\end{tabular}} & \multirow{4}{*}{$\begin{array}{l}\begin{array}{l}\text { Geometric } \\
\text { distance }\end{array} \\
1.1977228\end{array}$} & \multirow{4}{*}{$\begin{array}{l}\begin{array}{l}\text { Difference in } \\
\text { total density }\end{array} \\
65.42\end{array}$} & \multirow{4}{*}{$\begin{array}{l}\text { Density } \\
\text { / } \\
\text { distance }\end{array}$} \\
\hline & & \multirow{2}{*}{$\begin{array}{l}\text { longitude } \\
21.64455 \\
\end{array}$} & \multirow{2}{*}{$\begin{array}{l}\text { latitude } \\
-30.00486 \\
\end{array}$} & & & & \\
\hline 1 & 40 & & & & & & \\
\hline 1 & 42 & 22.83446 & -29.86828 & 101.83 & & & \\
\hline \multirow{3}{*}{2} & 101 & 18.20583 & -30.15250 & 70.00 & \multirow{3}{*}{$\begin{array}{l}0.285919122 \\
0.688683879 \\
0.787944636\end{array}$} & 6.67 & 23.33 \\
\hline & 102 & 17.92017 & -30.14033 & 63.33 & & 16.67 & 24.20 \\
\hline & 103 & 17.83583 & -29.45683 & 46.67 & & 23.33 & 29.61 \\
\hline \multirow{2}{*}{3} & 3 & 19.25749 & -27.49479 & 33.33 & \multirow[t]{2}{*}{0.003999625} & \multirow[t]{2}{*}{52.38} & \multirow[t]{2}{*}{13096.47} \\
\hline & 4 & 19.26062 & -27.49230 & 54.17 & & & \\
\hline
\end{tabular}

Table 1. 3 groups of sites and their total densities

The measure of habitat closeness can be defined by the habitat distance, which is a virtual distance indicating the aggregate ecological environment of an area. In such a circumstance, if we still impose conventional isotropic distance assumption to construct a kriging predictor, the prediction would be no sense at all.

It is logical to argue that in order to utilize the $n$ observations $\left\{Z(s), s \in \mathfrak{N}_{s_{0}} \subset \mathbb{R}^{2}\right\}$ contained in the neighbourhood of $s_{0}: \mathfrak{N}_{s_{0}}=\left\{s_{0}, s_{1}, s_{2}, \cdots, s_{n}\right\}$, at $s_{0}$ without an Quiver tree's observation, to predict $Z\left(s_{0}\right)$, utilize the habitat distance information is far more efficient that the conventional isotropic distance.

As to how to define the habitat distance measure, it depends upon the habitat spatial distribution pattern as well as the form of uncertainty governing the spatial process $\left\{Z(s), s \in \mathbb{R}^{2}\right\}$.

In this study, we are going to calculate habitat distance in following three approaches:

(1) Grouping method. This approach classifies total density observations $\left\{Z(s), s \in \mathbb{R}^{2}\right\}$ into groups by the sizes of $Z(s)$. Then, for each group, the variance is calculated. choose the variance of group observation as habitat measure. We may face the situation at which some $Z(s)$ values may be far away from those grouped observations. Then we may group them according to geometrical distance criterion, then calculate the variances for those isolated total density observations. Table 2 lists a few observational groups and group habitat distances.

It is logical to ignore those 3 groups because of extreme group variances, which should not accepted as habitat measures.

(2) Inverse-distance methods. Notice that there are ten sites without total density observations and thus their habitat measures cannot be determined. However, later we will see the empirical habitat measure is necessary for uncertain kriging.

In this paper, we propose two schemes for addressing the habitat measure at missing total density value site. The first one is the conventional inverse distance estimation. The second one is utilizing the empirical habitat distance for uncertain inverse distance estimation.

The conventional inverse distance estimation. Table 5 summarizes the results (at $\lambda=1.0$ because near optimal $\lambda=0.1$ gives illogical empirical results). 


\begin{tabular}{|c|c|c|c|c|c|}
\hline \multirow{2}{*}{\multicolumn{2}{|c|}{$\begin{array}{l}\text { Group/ } \\
\text { Site no. }\end{array}$}} & \multicolumn{2}{|c|}{ Coordinates } & \multirow{3}{*}{$\begin{array}{l}\text { Total density } \\
\text { of trees }\end{array}$} & \multirow{2}{*}{$\begin{array}{l}\text { Habitat } \\
\text { measure }\end{array}$} \\
\hline & & longitude & latitude & & \\
\hline \multirow{3}{*}{1} & 42 & 22.83446 & -29.86828 & & \multirow{3}{*}{1.1112310} \\
\hline & 11 & 18.18643 & -26.21253 & 100.00 & \\
\hline & 113 & 22.18500 & -29.06700 & 100.00 & \\
\hline \multirow{3}{*}{2} & 20 & 16.23986 & -25.59329 & 90.48 & \multirow{3}{*}{2.0052690} \\
\hline & 13 & 15.15805 & -21.48867 & 91.70 & \\
\hline & 124 & 19.43333 & -28.83333 & 93.30 & \\
\hline \multirow{4}{*}{3} & 4 & 19.26062 & -27.49230 & 33.33 & \multirow{4}{*}{3.4715580} \\
\hline & 2 & 19.28217 & -28.66671 & 33.33 & \\
\hline & 40 & 21.64455 & -30.00486 & 36.41 & \\
\hline & 123 & 19.40000 & -28.98333 & 36.70 & \\
\hline \multirow{3}{*}{4} & 104 & 17.28650 & -28.30430 & 40.00 & \multirow{3}{*}{11.1111222} \\
\hline & 108 & 19.45333 & -28.10333 & 43.33 & \\
\hline & 103 & 17.83583 & -29.45683 & 46.67 & \\
\hline \multirow{3}{*}{5} & 107 & 18.56950 & -27.68433 & 113.33 & \multirow{3}{*}{11.5226107} \\
\hline & 29 & 15.47427 & -26.58937 & 106.67 & \\
\hline & 30 & 16.76122 & -27.03209 & 111.11 & \\
\hline \multirow{4}{*}{6} & 41 & 22.37461 & -29.29727 & 86.05 & \multirow{4}{*}{11.5534900} \\
\hline & 121 & 19.43333 & -29.06667 & 80.00 & \\
\hline & 122 & 19.43333 & -29.06667 & 80.00 & \\
\hline & 3 & 19.25749 & -27.49479 & 85.71 & \\
\hline \multirow{3}{*}{7} & 112 & 22.37000 & -28.93500 & 130.00 & \multirow{3}{*}{14.9633000} \\
\hline & 126 & 21.10000 & -29.40000 & 123.30 & \\
\hline & 26 & 16.31455 & -27.64893 & 130.00 & \\
\hline \multirow{2}{*}{8} & 23 & 15.62385 & -26.99582 & 22.22 & \multirow{2}{*}{17.0993200} \\
\hline & 9 & 17.01403 & -26.66638 & 28.07 & \\
\hline \multirow{5}{*}{9} & 102 & 17.92017 & -30.14033 & 63.33 & \multirow{5}{*}{19.9113500} \\
\hline & 1 & 18.78301 & -28.71597 & 63.33 & \\
\hline & 101 & 18.20583 & -30.15250 & 70.00 & \\
\hline & 125 & 20.23333 & -28.61667 & 70.00 & \\
\hline & 120 & 18.83333 & -30.70000 & 73.30 & \\
\hline \multirow{3}{*}{10} & 110 & 18.98883 & -31.10050 & 50.00 & \multirow{3}{*}{21.5049200} \\
\hline & 5 & 19.59336 & -26.79313 & 54.17 & \\
\hline & 24 & 15.78774 & -27.31541 & 59.26 & \\
\hline \multirow{5}{*}{11} & 106 & 17.98333 & -26.40217 & 166.67 & \multirow{5}{*}{53.3333900} \\
\hline & 18 & 16.21400 & -23.95119 & 153.33 & \\
\hline & 105 & 18.23967 & -26.47550 & 153.33 & \\
\hline & 22 & 15.36674 & -26.88367 & 166.67 & \\
\hline & 28 & 16.14727 & -26.54360 & 166.67 & \\
\hline
\end{tabular}

Table 2. Groups of total densities and habitat measures (group variances) 
It is obvious that there are five sites left listed in Table 3.

\begin{tabular}{|l|l|l|l|l|l|}
\hline \multicolumn{2}{|l|}{$\begin{array}{l}\text { Group/ } \\
\text { site no. }\end{array}$} & Coordinates & Total density & Group variance \\
\cline { 2 - 5 } & of trees & \\
\hline 12 & 127 & 21.16667 & -29.08333 & 6.70 & \\
\hline 13 & 34 & 16.77862 & -27.64536 & 282.61 & \multirow{2}{*}{1843.7403440} \\
\cline { 2 - 5 } & 111 & 18.98567 & -31.10583 & 343.33 & \\
\hline \multirow{2}{*}{14} & 109 & 20.79000 & -28.74017 & 200.00 & \multirow{2}{*}{12482.0000000} \\
\cline { 2 - 5 } & 21 & 16.31081 & -25.88543 & 252.17 & \\
\hline
\end{tabular}

Table 3. 3 Groups of total densities left with group variances

\begin{tabular}{|l|l|l|l|l|}
\hline \multirow{2}{*}{ Site no. } & \multicolumn{2}{|l|}{ Coordinates } & $\begin{array}{l}\text { Predicted total } \\
\text { density of trees }\end{array}$ & $\begin{array}{l}\text { Predicted habitat } \\
\text { measure }\end{array}$ \\
\cline { 2 - 4 } & longitude & latitude & 103.2131 & 1.1112310 \\
\hline 15 & 14.57582 & -21.20986 & 102.9913 & 1.1112310 \\
\hline 14 & 14.63088 & -21.26169 & 102.3649 & 1.1112310 \\
\hline 12 & 15.21586 & -21.81786 & 110.5426 & 11.5226107 \\
\hline 17 & 15.51133 & -23.30893 & 115.2105 & 11.5226107 \\
\hline 19 & 16.39317 & -25.14609 & 121.9919 & 14.9633000 \\
\hline 33 & 16.77479 & -27.94278 & 106.7434 & 11.5226107 \\
\hline 36 & 17.47540 & -27.45209 & 110.3903 & 11.5226107 \\
\hline 10 & 17.78013 & -25.87191 & 101.0099 & 1.1112310 \\
\hline 37 & 17.80606 & -27.67034 & 82.67477 & 11.5534900 \\
\hline 128 & 21.10000 & -28.95000 & \\
\hline
\end{tabular}

Table 4. 10 Sites of Inverse Distance predicted total densities

From Table 4, we can see that since the estimated total density estimators are calculated in terms of graphical distances. Those estimators are empirical prior information. As to habitat measure, they are taking the values by comparing the estimated total density to the group total density values in Table 4. Definitely, the empirical habitat measures are not all accurate.

(3) Uncertain canonical process regression methods. Because the Quiver tree's total density is very sensitive to local area ecological environments, it is logical to consider if some uncertain relationship between the observed total density and ecological environmental variables, say, annual temperature, and annual rainfall. Table 4 lists the data, from which a linear relationship between group average total density $y_{d_{i}}$ and group average annual temperature $T_{d_{i}}$ and group average annual rainfall $R_{d_{i}}$ at the empirical distance $d_{i}, i=1,2, \cdots, 11$, is intended to establish.

The uncertain regression model takes a form:

$$
\begin{aligned}
& y_{d_{i}}=\alpha_{0}+\alpha_{1} T_{d_{i}}+\alpha_{2} R_{d_{i}}+\sigma C_{d_{i}}, \\
& i=1,2, \cdots, 11
\end{aligned}
$$


where errors $C_{d_{i}}, i=1,2, \cdots, 11$ are from the uncertain canonical process $\left\{C_{d}, d \in[0,+\infty)\right\}$. As to $\left\{C_{d}, d \in[0,+\infty)\right\}$. The fitted regression is

$$
\begin{aligned}
& \hat{y}_{d_{i}}=\underset{(66.6838)}{500.4016}+\underset{(6.1464)}{30.1100} T_{d_{i}}-\underset{(2.0621)}{8.8953} R_{d_{i}}, \\
& i=1,2, \cdots, 11
\end{aligned}
$$

\begin{tabular}{|c|c|c|c|c|}
\hline \multirow[t]{2}{*}{ Site no. } & \multicolumn{2}{|c|}{ Coordinates } & \multirow{2}{*}{$\begin{array}{l}\text { Predicted total } \\
\text { density of trees }\end{array}$} & \multirow{2}{*}{$\begin{array}{l}\text { Predicted habitat } \\
\text { measure }\end{array}$} \\
\hline & longitude & latitude & & \\
\hline 15 & 14.57582 & -21.20986 & 110.3210 & 11.5226 \\
\hline 14 & 14.63088 & -21.26169 & 26.5995 & 17.0993 \\
\hline 12 & 15.21586 & -21.81786 & 55.0573 & 21.5049 \\
\hline 17 & 15.51133 & -23.30893 & 83.5152 & 11.5535 \\
\hline 19 & 16.39317 & -25.14609 & 8.72890 & 2.0582 \\
\hline 33 & 16.77479 & -27.94278 & 147.0394 & 53.3334 \\
\hline 36 & 17.47540 & -27.45209 & 72.2531 & 19.9114 \\
\hline 10 & 17.78013 & -25.87191 & 20.9684 & 17.0993 \\
\hline 37 & 17.80606 & -27.67034 & 29.9036 & 17.0993 \\
\hline 128 & 21.10000 & -28.95000 & 101.3857 & 1.1112 \\
\hline
\end{tabular}

Table 5. 10 Sites of Regression Predicted total densities left with habitat measures

However, it is necessary to mention that the uncertain regression model is carried on the group average total density against group average annual temperature and group annual rainfall and thus the predictability is limited although the model goodness-of fit $\left(R^{2} \approx 0.7\right)$ is far better than that of classical regression model $\left(R^{2} \approx 0.21\right)$, whose coefficients for annual temperature and annual rainfall are both insignificant statistically. We are not going to use these regression predicted total density of trees as the basic results for evaluations of climate change impacts.

Finally, we must emphasize that for any given spatial process $\left\{Z(s), s \in \mathbb{R}^{2}\right\}$, which has no order, this fact would prevent geostatistical analysts from utilizing 1-dimensional statistical approaches for predictions, for example, the uncertain regression engaged. But after defining appropriate habitat distance for spatial process, then the habitat distance set can be indexed an uncertain canonical process as the desired partner process, and hence the intrinsic covariance structure of the partner process will be available and thus simplify greatly the semi-variogram computations, while in probabilistic kriging the theoretical semivariogram must be replaced by sample or experimental semi-variogram. The uncertain semi-varogram is semi-data oriented, in contrast, the probabilistic sample or experimental semi-variogram is fully data-oriented.

\section{Uncertain kriging predictor}

We first point out that kriging predictor is not intrinsic to the probabilistic spatial statistics. As long as variance, covariance, and semi-variogram concepts can be established on some uncertain theory, no matter it is Zadeh's fuzzy theory, rough set theory, grey theory, random set theory, or interval theory, or Liu's uncertainty theory, new kriging predictor can 
be solidly established with similar mathematical formulae as that of probabilistic ordinary kriging (Cressie, 1991). However, the geometric location s should be replaced by habitat distance $d(s)$, which is the square root of habitat measure.

An uncertain semi-variogram is defined by

$$
2 \gamma\left(d_{s_{i}}-d_{s_{j}}\right)=V\left(Z\left(d_{s_{i}}\right)-Z\left(d_{s_{j}}\right)\right)
$$

where $d_{s_{i}}$ and $d_{s_{i}}$ are two habitat distances at location $s_{i}$ and location $s_{j}$ respectively. The uncertain kriging predictor is defined by

$$
Z\left(d_{s_{0}}\right)=\sum_{i=1}^{n} \lambda_{i} Z\left(d_{s_{i}}\right)
$$

where $\lambda_{1}, \lambda_{2}, \cdots, \lambda_{n}$ are determined by minimizing the following objective function.

$$
\mathrm{E}\left[\left(Z\left(d_{s_{0}}\right)-\sum_{i=1}^{n} \lambda_{i} Z\left(d_{s_{i}}\right)\right)^{2}\right]-2 \psi\left(\sum_{i=1}^{n} \lambda_{i}-1\right)
$$

where $\psi$ is the Lagrange's multiplier. Then the uncertain kriging equation system is given by

$$
\underline{\lambda}_{U}=\Gamma_{U}^{-1} \underline{\gamma}_{U}
$$

where

$$
\begin{aligned}
& \underline{\lambda}_{U}=\left(\lambda_{1}, \lambda_{2}, \cdots, \lambda_{n}, \psi\right)^{\prime}, \\
& \underline{\gamma}_{U}=\left(\gamma\left(d_{s_{1}}-d_{s_{0}}\right), \gamma\left(d_{s_{2}}-d_{s_{0}}\right), \cdots, \gamma\left(d_{s_{n}}-d_{s_{0}}\right), 1\right)^{\prime} \\
& \Gamma_{U}=\left(o_{i j}\right)_{(n+1) \times(n+1)} \\
& o_{i j}=\left\{\begin{array}{cc}
\sigma^{2} & i=j=1,2, \cdots, n \\
\gamma\left(d_{s_{i}}-d_{s_{j}}\right) & i \neq j=1,2, \cdots, n \\
1 & i=n+1, j=1,2, \cdots, n \\
0 & i=j=n+1
\end{array}\right.
\end{aligned}
$$

Theorem 5.1: Let

$$
\underline{\lambda}=\left(\lambda_{1}, \lambda_{2}, \cdots, \lambda_{n}\right)^{\prime}
$$

then,

$$
\underline{\lambda}^{\prime}=\left(\underline{\gamma}+\underline{1} \frac{\left(1-\underline{1}^{\prime} \Gamma^{-1} \underline{\gamma}\right)}{\underline{1}^{\prime} \Gamma^{-1} \underline{1}}\right) \Gamma^{-1}
$$


where

$$
\psi=-\frac{\left(1-\underline{1}^{\prime} \Gamma^{-1} \underline{\gamma}\right)}{\underline{1}^{\prime} \Gamma^{-1} \underline{1}}
$$

Theorem 5.2: Let $d_{s_{i}, s_{j}} \triangleq d_{s_{j}}-d_{s_{i}}, d_{s_{i}} \leq d_{s_{j}}$. Then the semi-variogram of habitat distance $d_{s_{i}, s_{j}}$ is

$$
\gamma\left(d_{s_{i}, s_{j}}\right)=\frac{1}{2}\left(d_{s_{j}}-d_{s_{i}}\right)-\delta_{d_{s_{i}}, d_{s_{j}}-d_{s_{i}}}
$$

Proof: It is noticed that for $\forall d_{s_{i}} \leq d_{s_{j}}$

$$
\begin{aligned}
\gamma\left(d_{s_{i}, s_{j}}\right) & \\
= & \frac{1}{2} \mathrm{E}\left[\left(Z\left(d_{s_{i}}\right)-Z\left(d_{s_{j}}\right)\right)^{2}\right] \\
= & \frac{1}{2} \mathrm{E}\left[\left(\left(Z\left(d_{s_{i}}\right)-m\right)-\left(Z\left(d_{s_{j}}\right)-m\right)\right)^{2}\right] \\
= & \frac{1}{2} \mathrm{E}\left[\left(Z\left(d_{s_{i}}\right)-m\right)^{2}\right]+\frac{1}{2} \mathrm{E}\left[\left(Z\left(d_{s_{j}}\right)-m\right)^{2}\right] \\
- & \mathrm{E}\left[\left(Z\left(d_{s_{i}}\right)-m\right)\left(Z\left(d_{s_{j}}\right)-m\right)\right] \\
= & \frac{1}{2}\left(\sigma^{2}\left(Z\left(s_{i}\right)\right)+\sigma^{2}\left(Z\left(s_{j}\right)\right)\right)-\mathrm{E}\left[\varepsilon\left(d_{s_{i}}\right) \varepsilon\left(d_{s_{j}}\right)\right] \\
= & \frac{1}{2}\left(d_{s_{i}}+d_{s_{j}}\right)-\sigma_{d_{s_{i}}}, d_{s_{j}} \\
= & \frac{1}{2}\left(d_{s_{i}}+d_{s_{j}}\right)-\left(d_{s_{i}}+\delta_{d_{s_{i}}}, d_{s_{j}}-d_{s_{i}}\right) \\
= & \frac{1}{2}\left(d_{s_{j}}-d_{s_{i}}\right)-\delta_{d_{s_{i}}, d_{s_{j}}-d_{s_{i}}}
\end{aligned}
$$

However, at location $s_{0}$, there is no observation $Z\left(s_{0}\right)$ and thus the habitat distance $d_{s_{0}}$ is undefined. It is necessary to "define" the habitat distance $d_{s_{0}}$ in order to carry on the uncertain kriging predictions.

Definition 5.3: If geometric location $s_{k}$ is close to $s_{0}$, at which $Z\left(s_{k}\right)$ is observed, then the habitat distance of $Z\left(s_{k}\right)$ is defined as the habitat distance $d_{s_{0}}$ at location $s_{0}$.

There is possibility that some $\lambda_{i_{n p}}<0$. Sort $\left\{\lambda_{1}, \lambda_{2}, \cdots, \lambda_{n}\right\}$ such that $\lambda_{(1)} \leq \lambda_{(2)} \leq \cdots \leq \lambda_{(n)}$, assuming $i_{p}: \lambda_{\left(i_{p}-1\right)}<0, \lambda_{\left(i_{p}\right)}>0$ e that there are $n_{n p}$ terms which but $\sum_{i=1}^{n_{s_{0}}} \lambda_{(i)}=1$, but $\sum_{i=1}^{n_{s_{0}}}\left|\lambda_{(i)}\right|>1$. Let us define the adjusted coefficient $\lambda_{i}^{\text {adjusted }}$ as 


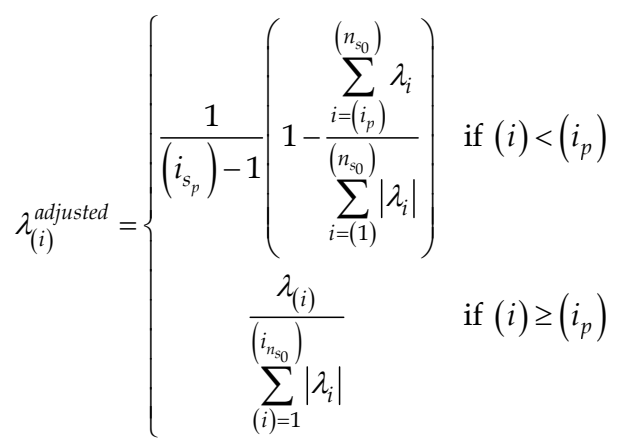

It is obvious that constraint

$$
\sum_{(i)=1}^{\left(n_{s_{0}}\right)} \lambda_{(i)}^{\text {adjusted }}=1.0
$$

is truly kept and also all $\lambda_{i}^{\text {adjusted }} \in(0,1)$.

We will calculate those uncertain kriging predictors for the ten sites $\{10,12,14,15,17,19,33,36$, $37,128\}$ without total density observations in following 4 steps.

Step 1: Determine the 10 neighbourhoods

$$
\left\{\mathcal{N}_{s_{0}^{(k)}}\right\}_{k=1}^{10}
$$

where $s_{0}^{(k)} \in\{10,12,14,15,17,19,33,36,37,128\}$. Each $\mathcal{N}_{s_{0}^{(k)}}$ contains six sites with observed total density values.

Step 2: For each individual neighbourhood $\mathcal{N}_{s_{0}^{(k)}}$, for example, $\mathcal{N}_{s_{0}^{(k)}}=\left\{s_{1}^{(k)}, s_{2}^{(k)}, \cdots, s_{6}^{(k)}\right\}$, determine the corresponding habitat distance from Table 3, 4, and 6, respectively, denoted by

$$
\mathcal{D}^{(k)}=\left\{d_{s_{1}^{(k)}}, d_{s_{2}^{(k)}}, \cdots, d s_{(k)}, d_{s_{0}^{(k)}}\right\}
$$

Step 3: Calculate those uncertain semi-variograms

$$
\begin{gathered}
\gamma\left(d_{s_{i}^{(k)}, s_{j}^{(k)}}\right), \forall d_{s_{i}^{(k)}}<d_{s_{j}^{(k)}} \text { and } \\
\gamma\left(d_{s_{0}^{(k)}, s_{i}^{(k)}}\right), \forall d_{s_{0}^{(k)}}<d_{s_{i}^{(k)}}\left(\text { or } \gamma\left(d_{s_{i}^{(k)}, s_{0}^{(k)}}\right), \forall d_{s_{i}^{(k)}}<d_{s_{0}^{(k)}}\right)
\end{gathered}
$$

in terms of

$$
\gamma\left(d_{s_{i}^{(k)}, s_{j}^{(k)}}\right)=\frac{1}{2}\left(d_{s_{j}^{(k)}}-d_{s_{i}^{(k)}}\right)-\delta_{d_{s_{i}^{(k)}}, d_{s_{j}}-d_{s_{i}}}
$$


with

$$
\begin{aligned}
& \delta_{d_{s_{i}^{(k)}}, d_{s_{j}^{(k)}}-d_{s_{i}^{(k)}}} \\
& =\int_{0}^{\infty}\left(1-\sup _{x y=r}\left(\Psi_{C_{d_{s}(k)}}(x) \wedge \Psi_{C_{d_{i}(k)-d} s_{j}(k)}(y)\right)\right) d r \\
& -\int_{-\infty}^{0} \sup _{x y=r}\left(\Psi_{C_{d_{s}(k)}}(x) \wedge \Psi_{C_{d_{i}(k)-d}(k)}(y)\right) d r,
\end{aligned}
$$

Step 4: Calculate $\left\{\lambda_{1}^{(k)}, \cdots, \lambda_{6}^{(k)}\right\}$ according to equation (25).

Step 5: Calculate the kriging predictor

$$
Z\left(s_{0}^{(k)}\right)=\sum_{i=1}^{n^{(k)}} \lambda_{i}^{(k)} Z\left(s_{i}^{(k)}\right)
$$

The upper bound $1 \leq n^{(k)} \leq 6$, in case of $n^{(k)}<6$, which implies some locations have the same habitat distance value, we simply put the average of total density values with the same habitat distance to participate the uncertain kriging predictor computations.

Step 6: Repeat Step 2 to Step 5, until all 10 uncertain kriging predictors are calculated. Then stop.

Table 7 summarizes uncertain kriging predictor values, and lists the no total density observation sties (similar to Table 6).

\begin{tabular}{|l|l|l|l|}
\hline \multirow{2}{*}{ site no. } & \multicolumn{2}{|l|}{ Coordinates } & Uncertain kriging predicted \\
\cline { 2 - 3 } & longitude & latitude & total density of trees \\
\hline 15 & 14.57582 & -21.20986 & 132.446 \\
\hline 14 & 14.63088 & -21.26169 & 155.987 \\
\hline 12 & 15.21586 & -21.81786 & 177.641 \\
\hline 17 & 15.51133 & -23.30893 & 132.567 \\
\hline 19 & 16.39317 & -25.14609 & 99.266 \\
\hline 33 & 16.77479 & -27.94278 & 62.493 \\
\hline 36 & 17.47540 & -27.45209 & 117.045 \\
\hline 10 & 17.78013 & -25.87191 & 121.982 \\
\hline 37 & 17.80606 & -27.67034 & 72.249 \\
\hline 128 & 21.10000 & -28.95000 & 44.087 \\
\hline
\end{tabular}

Table 6. Uncertain Kriging Predictions for 10 sites

Next, we will use uncertain kriging methods to predict the missing values for the population growth rate. There are 18 sites without values: Site number $\{120,111,110,125,22,23,24,26,123,124,121,122,17,34,126,128,127,42\}$ 


\begin{tabular}{|l|l|l|l|l|}
\hline \multirow{2}{*}{ site no. } & Coordinates & Habitat \\
\cline { 2 - 4 } & longitude & latitude & $\begin{array}{l}\text { Uncertain kriging } \\
\text { Predicted population } \\
\text { growth rate }\end{array}$ \\
\hline 120 & 18.83333 & -30.7 & 19.91135 & 1.02490171 \\
\hline 111 & 18.98567 & -31.1058 & 1843.74 & 1.027150902 \\
\hline 110 & 18.98883 & -31.1005 & 21.50492 & 1.025065854 \\
\hline 125 & 20.23333 & -28.6167 & 19.91135 & 1.01815189 \\
\hline 22 & 15.36674 & -26.8837 & 53.33339 & 0.995053254 \\
\hline 23 & 15.62385 & -26.9958 & 17.09932 & 1.030192199 \\
\hline 24 & 15.78774 & -27.3154 & 21.50492 & 1.036620495 \\
\hline 26 & 16.31455 & -27.6489 & 14.9633 & 1.006659104 \\
\hline 123 & 19.4 & -28.9833 & 3.471558 & 1.001714497 \\
\hline 121 & 19.43333 & -28.8333 & 2.005269 & 1.004648936 \\
\hline 124 & 19.43333 & -29.0667 & 11.55349 & 1.014237773 \\
\hline 122 & 19.43333 & -29.0667 & 11.55349 & 1.014237773 \\
\hline 17 & 15.51133 & -23.3089 & 11.5535 & 0.999365585 \\
\hline 34 & 16.77862 & -27.6454 & 1843.74 & 1.000142274 \\
\hline 126 & 21.1 & -29.4 & 14.9633 & 1.02687582 \\
\hline 128 & 21.1 & -28.95 & 1.1112 & 1.014980439 \\
\hline 127 & 21.16667 & -29.0833 & 2.0582 & 1.005842705 \\
\hline 42 & 22.83446 & -29.8683 & 1.111231 & 1.003521691 \\
\hline
\end{tabular}

Table 7. Uncertain Kriging Predictions for 18 sites

\section{Climate change impacts}

Now we are ready to calculate the missing values of 2008 total density of trees, which are 25 sites. We utilize the compound growth rate formula:

$$
\kappa_{2008}^{(k)}=\kappa_{2002}^{(k)}\left(1+r^{(k)}\right)^{6}
$$

Denote the Site $k$ population growth rate during $i$ years' period as $\rho_{(i)}^{(k)}$, then, we can use the compound growth formula,

$$
\rho_{(i)}^{(k)}=\left(1+r^{(k)}\right)^{i}
$$

Then

$$
r^{(k)}=\exp \left(\frac{1}{i} \ln \left(\rho_{(i)}^{(k)}\right)\right)-1
$$

Therefore, if the total density $\kappa_{2002}^{(k)}$ is available, no matter it is observed or predicted in terms of uncertain kriging approach, $\kappa_{2008}^{(k)}$ will be determined.

After the calculations, we have complete data of $\kappa_{2008}^{(k)}$ and $\kappa_{2008}^{(k)}$ for site $k$, then The change in site $k$ will be 


$$
\delta^{(k)}=\kappa_{2008}^{(k)}-\kappa_{2002}^{(k)}
$$

The final uncertain predicted results together with the original sampled results of 2002 and 2008 are shown in the Fig. 3. The reason that they look exactly the same with absolutely no difference at all, is because the increase and decrease in total densities are so small, that they cannot be viewed just by looking at the intervals.
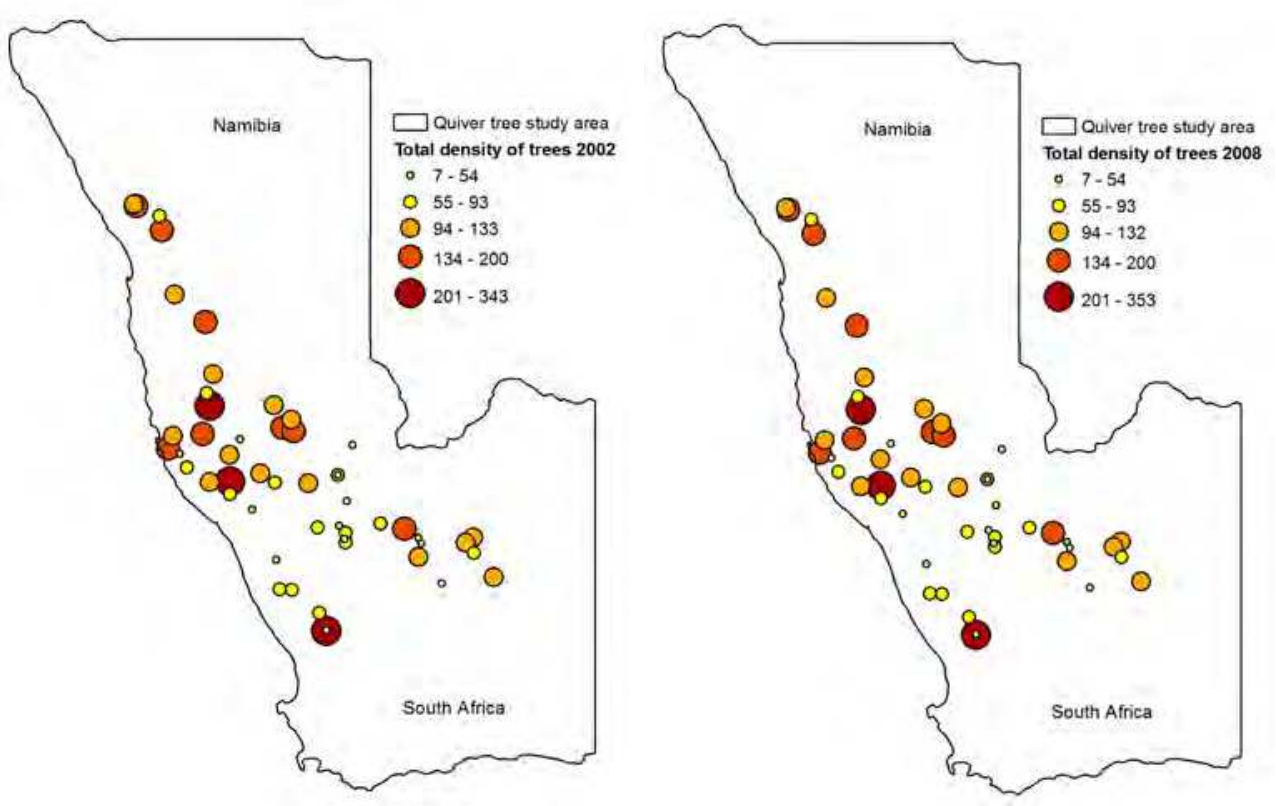

Fig. 3. Sampled and Predicted total density of Quiver trees in Namibia and South Africa, 2002 and 2008

In Fig. 4 the Ordinary kriging prediction maps of sampled and predicted total density of Quiver trees in 2002 and 2008 are shown. Since the population increase and decrease are very small, which is why they are only reflected using kriging maps. However, the kriging maps are slightly distorted in value, due to re-calculations of sample and predicted values, and the average mean values are used.

Table 8 and Fig. 5 show the change in the total density of Quiver trees over a 6 year period, between 2002 and 2008 .

\begin{tabular}{|l|l|l|l|}
\hline & $\begin{array}{l}\text { Negative change } \\
\left(\delta^{(k)}<0\right)\end{array}$ & $\begin{array}{l}\text { Positive change } \\
\left(\delta^{(k)}>0\right)\end{array}$ & $\begin{array}{l}\text { unchanged } \\
\left(\delta^{(k)}=0\right)\end{array}$ \\
\hline No. Of sites & 7 & 18 & 28 \\
\hline Percentages & $13.21 \%$ & $33.96 \%$ & $52.83 \%$ \\
\hline
\end{tabular}

Table 8. Climate change impact on total density of Quiver trees over 6 year period 


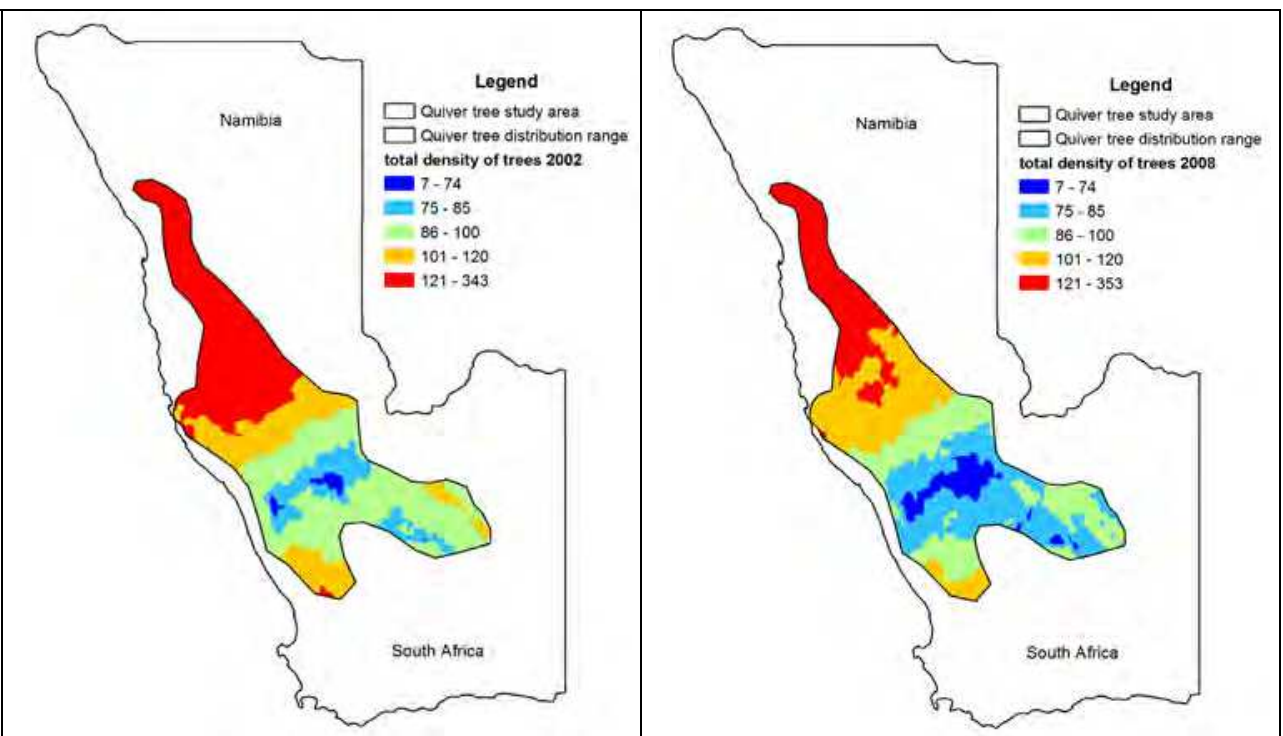

Fig. 4. Ordinary Kriging prediction maps of the total density of Quiver trees, 2002 and 2008

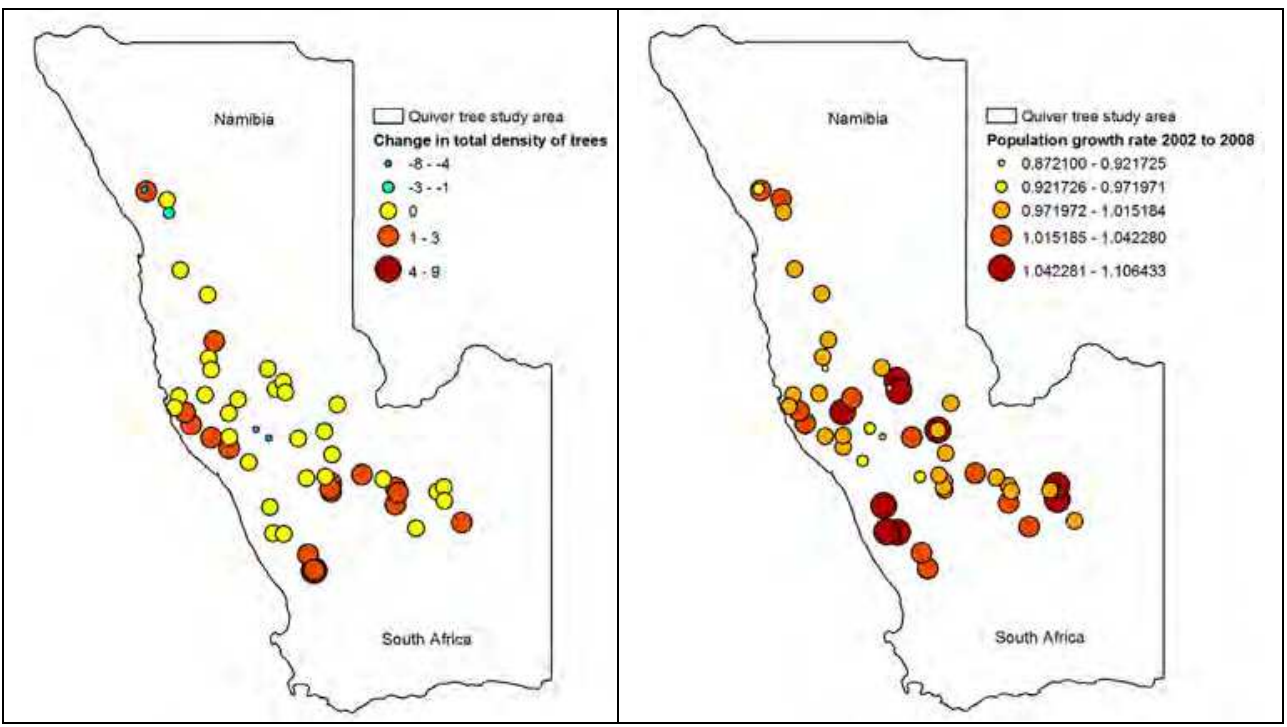

Fig. 5. Predicted change in total density of trees, and calculated population growth of Quiver trees, between 2002 and 2008

As one can see from the Fig. 5 change map, the change in total density of trees is very small. Most sample sites show no changes at all, with 18 sites show an increase of 1 to 9 trees, and 7 sites showing a decrease of 1 to 8 trees. These are small but definite changes in Quiver tree's total density over the 6 year period, between 2002 and 2008. 


\section{Conclusion}

In this paper, we utilize the partially collected Quiver tree's sample data, 2002 and 2008 datasets, particularly, the total density of trees to evaluate the climate change impact on the Quiver tree's population. Because the data collection sites are only 53 and the Quiver tree's population size is extremely sensitive to it habitat environment, the conventional methodologies are not applicable. Based on our observations, we propose the habitat measure to catch up the closeness of the Quiver tree's population, which is later defining the habit distance of the tree's population. The habitat distance set is then regarded as the partner process index set. Therefore, we are able to engage Liu's Uncertainty theory for developing a new uncertain kriging approach to facilitate the prediction task: utilizing "complete" (53 values) data to perform the change evaluation. Once the missing values are filled, the evaluation of climate change can be performed. We are aware that our new prediction results have not had a validation process because of the time-constraint. In the future, it is necessary to engage the model accuracy and validation checking. Overall, there is a small but definite change in Quiver trees over the 6 year period, between 2002 and 2008, possibly due to climate changes over time.

Of course, monitoring the Quiver trees and using it as a climate change indicator, is a continuing process and effort for the South African National Biodiversity Institute. The results from this paper help to complete the missing data or un-sampled data, and would be useful for future comparisons, when another sample collection is made. The uncertain prediction methods and calculation process may be useful with other kinds of plant species data that displays similar problems, such as missing sample values. The predicted values together with the real sample values could be very useful in examining climate change impact over time and for studying the comparisons of plant species from different periods.

\section{Acknowledgements}

Sincere thanks to Wendy Foden and Graeme Ellis for their contribution to Aloe dichotoma data collections, and to the South African National Biodiversity Institute for their support of the Aloe Project. This book chapter is supported financially by the National Research Foundation of South Africa (Ref. No. IFR2009090800013) and (Ref. No. IFR2011040400096).

\section{References}

Bardossy, A.; Bogardi, I. \& Kelly, E. (1990). Kriging with imprecise (fuzzy) variograms, I: Theory. Mathematical Geology, Vol. 22, pp. 63-79.

Cressie, N. (1991). Statistics for Spatial Data. Wiley-Interscience, John-Wiley \& Sons Inc. New York.

Deng, J.L. (1984). Grey dynamic modeling and its application in long-term prediction of food productions. Exploration of Nature, Vol. 3, No. 3, pp. 7-43.

Draper, N. \& Smith, H. (1981). Applied Regression Analysis. 2nd Edition. John Wiley \& Sons, Inc. New York.

Foden, W. (2002). A Demographic Study of Aloe dichotoma in the Succulent Karoo: Are the Effects of Climate Change Already Apparent? MSc Thesis, Percy FitzPatrick Institute of African Ornithology, Botany Department, University of Cape Town. 
Guo, D. (2010). Contributions to Spatial Uncertainty Modelling in GIS. Lambert Academic Publishing (online.lap-publishing.com). ISBN 978-3-8433-7388-3

Guo, D.; Guo, R.; Midgley, G.F. \& Rebelo, A.G. (2007). PDEMR Modelling of Protea Species in the Population Size of 1 to 10, in Cape Floristic Region from 1992 to 2002, South Africa. Journal of Geographic Information Sciences, Vol. 12, No. 2, pp. 67-78.

Midgley, G.F.; Altwegg, R.; Guo, D. \& Merow, C. (2009). Are Quiver Trees a Sentinel for Climate Change in Arid Southern Africa? South African National Biodiversity Institute. ISBN: 978-0-620-43639-7

Kolmogorov, A.N. (1950) Foundations of the Theory of Probability. Translated by Nathan Morrison. Chelsea, New York.

Liu, B.D. (2007). Uncertainty Theory: An Introduction to Its Axiomatic Foundations. 2nd Edition. Springer-Verlag Heidelberg, Berlin.

Liu, B.D. (2010). Uncertainty Theory: A Branch of Mathematics of Modelling Human Uncertainty. Springer-Verlag, Berlin.

Liu, B.D. (2011). Uncertainty Theory, 4th Edition, 17 February, 2011 drafted version.

Liu, S.F. \& Lin, Y. (2006). Grey Information. Springer-Verlag, London.

Mase, S. (2011). GeoStatistics and Kriging Predictors, In: International Encyclopedia of Statistical Science. Editor: Miodrag Lovric, 1st Edition, 2011, LVIII, pp. 609-612, Springer.

Moore, R.E. (1966). Interval Analysis. Prentice-Hall, Englewood Cliff, NJ. ISBN 0-13-476853-1

Pawlak, Z. (1982). Rough Sets. International Journal of Computer and Information Sciences, Vol. 11, pp. 341-356.

Utkin, L.V. \& Gurov, S.V. (1998). New reliability models on the basis of the theory of imprecise probabilities. Proceedings of the $5^{\text {th }}$ International Conference on Soft Computing and Information Intelligent Systems, Vol. 2, pp. 656-659.

Utkin, L.V. \& Gurov, S.V. (2000). New Reliability Models Based on Imprecise Probabilities. Advanced Signal Processing Technology, Soft Computing. Fuzzy Logic Systems Institute (FLSI) Soft Computing Series - Vol. 1, pp. 110-139, Charles Hsu (editor). Publisher, World Scientific. November 2000. ISBN 9789812792105

Van Wyk, B.E. \& Smith, G. (1996). Guide to Aloes of South Africa. Pretoria, Briza Publications.

Walley, P. (1991). Statistical Reasoning with Imprecise Probabilities. London: Chapman and Hall. ISBN 0412286602

Zadeh, L. A. (1965). Fuzzy sets. Information and Control, Vol. 8, pp. 338-353.

Zadeh, L. A. (1978). Fuzzy sets as a basis for a theory of possibility. Fuzzy Sets and Systems, Vol. 1, pp. 3-28. 


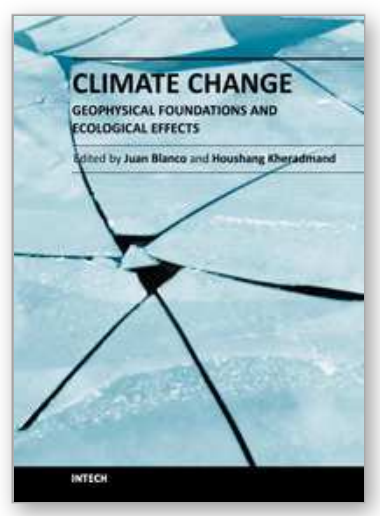

\author{
Climate Change - Geophysical Foundations and Ecological Effects \\ Edited by Dr Juan Blanco
}

ISBN 978-953-307-419-1

Hard cover, 520 pages

Publisher InTech

Published online 12, September, 2011

Published in print edition September, 2011

This book offers an interdisciplinary view of the biophysical issues related to climate change. Climate change is a phenomenon by which the long-term averages of weather events (i.e. temperature, precipitation, wind speed, etc.) that define the climate of a region are not constant but change over time. There have been a series of past periods of climatic change, registered in historical or paleoecological records. In the first section of this book, a series of state-of-the-art research projects explore the biophysical causes for climate change and the techniques currently being used and developed for its detection in several regions of the world. The second section of the book explores the effects that have been reported already on the flora and fauna in different ecosystems around the globe. Among them, the ecosystems and landscapes in arctic and alpine regions are expected to be among the most affected by the change in climate, as they will suffer the more intense changes. The final section of this book explores in detail those issues.

\title{
How to reference
}

In order to correctly reference this scholarly work, feel free to copy and paste the following:

Danni Guo, Renkuan Guo, Yanhong Cui, Guy F. Midgley, Res Altwegg and Christien Thiart (2011). Climate Change Impact on Quiver Trees in Arid Namibia and South Africa, Climate Change - Geophysical Foundations and Ecological Effects, Dr Juan Blanco (Ed.), ISBN: 978-953-307-419-1, InTech, Available from:

http://www.intechopen.com/books/climate-change-geophysical-foundations-and-ecological-effects/climatechange-impact-on-quiver-trees-in-arid-namibia-and-south-africa

\section{INTECH}

open science | open minds

\section{InTech Europe}

University Campus STeP Ri

Slavka Krautzeka 83/A

51000 Rijeka, Croatia

Phone: +385 (51) 770447

Fax: +385 (51) 686166

www.intechopen.com

\section{InTech China}

Unit 405, Office Block, Hotel Equatorial Shanghai

No.65, Yan An Road (West), Shanghai, 200040, China

中国上海市延安西路65号上海国际贵都大饭店办公楼405单元

Phone: +86-21-62489820

Fax: $+86-21-62489821$ 
(C) 2011 The Author(s). Licensee IntechOpen. This chapter is distributed under the terms of the Creative Commons Attribution-NonCommercialShareAlike-3.0 License, which permits use, distribution and reproduction for non-commercial purposes, provided the original is properly cited and derivative works building on this content are distributed under the same license. 